the unsatisfactory results obtained. A careful study of the different steps in the formation of an inguinal hernia will indicate the only method by which the injury can be repaired. The first step is the bulging or giving way of the parietal peritoneum, the sac of the hernia. Unless checked this gradually insinuates itself into the opening in the transversalis fascia forming the internal ring, causing a gaping or pouting of the lower lip. The heruial tumor then forces its way through the canal displacing the tissues of which it is composed, particularly the outer wall of the canal, and finally appears at the external ring.

The indications to be met to insure success are: first, the restoration of the peritoneum to its normal condition as near as possible, so as to permit of the free gliding movement of the bowels over the surface. To accomplish this the sac should be freed from all adhesions, the peritoneum loosened for some distance around the internal ring and gently pulled down, aud the whole length of the sac should then be cut away, not only to obliterate the depression of its surface, but to remove every portion of the peritoneum which has become more or less altered from stretching. For the same reason the cut surfaces should be sutured together so as to present as smooth a surface as possible. When tied in a ligature there is more or less puckering which resists the free movements of the bowels over its surface; second, the pouting of the lower lip of the internal ring should be obliterated so as no longer to invite intrusion of the bowel, while the displaced external wall of the canal should be approximated to the inner wall, the better to support the peritoneum and transversalis fascia. This can be accomplished by passing two sutures parallel with the outer line of the canal as in the Macewen operation, the upper one including the Iower edge of the internal ring. In large herniæ where the whole external wall is displaced, it would be well to insert two sutures in the same way along the inner line of the canal. In large old herniæ, where the internal ring is much dilated so as to be opposite the external ring, it will be necessary to freshen the hardened edges of the ring and suture them together with a continuous double suture. Every step of the operation should be done under the strictest antisepsis; all dead pockets should be avoided as they serve only to collect blood and serum; drainage tubes should not be used, if possible, and every effort should be made to secure healing by first intention, as it materially increases the prospects of success.

To be effectual and permanent, therefore, the operation must produce obliteration of the hernial sac, closure of its ventral orifice and strengthening of those weak parts in the abdominal parietes through which the rupture protrudes. At the same time, measures must be taken to produce an improved tone of the general system, particularly of the peritoneal ligaments of the viscera, by which the power they exert in retaining the organs in their normal position is restored. The more this is accomplished, and the closer the processes of nature are imitated, the more perfect the result; but no operation can be considered a "radical cure" that does not enable the sufferer to dispense with the use of a truss.

Hospital Closed. - The Binghampton (N. Y.) City Hospital has been closed, the Common Council having refused to vote the money for its support.

\section{STRANGULATED HERNIA.}

Read at the Meeting of the Inter-County Medical Society, held at West superior, Wis., July 11, 1893.

BY WILLIAM E. GROUND, M.D.

SUPERIOR, wIS.

I would like to consider with you to-day the subject of strangulated hernia. Probably the two most important surgical subjects before the profession just how are appendicitis and hernia. Every cemetery contains the graves of patients who have unnecessarily died from these affections.

The frequency of hernia varies somewhat at the different periods of life. It is met with very frequently during infancy. During early life it is generally of the congenital inguinal variety and is more common on the right than on the left side, probably owing to the fact that the root of the mesentery occupies a lower position on the right side. The greater proportionate length of the mesentery in infancy greatly favors the occurrence of hernial protrusions of the intestines during that period of life. Mr. Lockwood has shown that the mesentery in the infant measures one-fifth the length of the body, at puberty one-eighth and in adult life one-ninth. After early life the frequency of hernia diminishes rapidly, until after puberty. From puberty the number grows larger as age advances until at the age of sixty, it has been said that one in four or five are ruptured. Taking the human race as a whole, it has been estimated by surgeons of large experience that from one-sixteenth to one-eighth are afflicted with this complaint. While it is generally admitted that hernia occurs most frequently in the laboring classes, there are many conditions in those of more sedentary habits which must necessarily favor the escape of the various abdominal viscera.

Among the factors operating and in a general way favoring hernia may be mentioned heredity. Not that there is any direct transmission but rather the conditions that predispose to it, viz., the weakness of abdominal wall, openness of the rings, long mesentery. The stretching of the abdominal walls by pregnancies render lax the pelvic peritoneum during the child bearing period, and thus greatly favors the occurrence of femoral hernia at that time of life. The increase of volume and weight of the omentum by the deposit of fat favor the occurrence of hernia. Weakening and debilitating diseases cause the tissues to become relaxed and in that way encourage hernia.

Certain necessary anatomical conditions or defects in the abdominal wall renders hernia possible. They may be natural weaknesses or exaggerations of the natural, if I may be allowed the use of so far-fetched an expression, or the retentive powers of the abdom. inal walls may be diminished by traumatisms, either intentional or accidental. Men have larger inguinal rings; women have a larger femoral arch, and one less filled in by muscles and less strong fascia.

Conditions being favorable certain immediate influences may result in the production of a hernia. These are lifting, coughing, crying, and straining to expel urine or feces.

A hernia is generally enclosed in a sac consisting of a protruded portion of the peritoneum. The peritoneal protrusion may have been pushed gradually through one of the openings as in the acquired inguinal form, or the process carried down by the 
descent of the testicle may remain patent, so that the tunica vaginalis may form the sac in the congenital variety.

Femoral hernia is never congenital.

In inguinal hernia the parts that immediately concern us are the external and internal abdominal rings, and the inguinal canal. Still, it must be remembered at the outset that the rings and canal are only potential-they do not exist as rings or canal save when opened by a protrusion of the abdominal viscera or made so by a scalpel.

The separation or gap in the aponeurosis of the external oblique, called the external ring, allows the exit of the testicle and the spermatic cord in the male, and the round ligament of the uterus in the female. The size of the opening and the development and strength of its pillars and the fascia covering it vary greatly.

The internal ring is merely a funnel shaped expansion of the transverse fascia which the cord car. ries on with it as it escapes from the abdomen.

The flat sided passage in the abdominal wall between the external and internal rings is the inguinal canal. In very early life there is no canal, one ring lying directly behind the others to facilitate the passage of the testis. In the adult it measures about one inch and a half in length. The increased obliquity gives additional security.

A fullness or swelling out at one of the weak spots of the abdominal wall, which is more evident on standing, and which is especially noticeable when coughing or lifting, with the thighs flexed, is characteristic of the first appearance of hernia. It is a smooth uniform swelling which usually disappears when lying down to again reappear on standing. When the patient coughs or strains the tumor becomes tense, rises under the hand and expands in size. This manifestation is called the impulse and is generally characteristic of non-strangulated hernia.

Every organ in the abdomen has been known to have been herniated except the pancreas. The most common contents, however, of a hernial sac is some part of the intestine, usually the small bowel, or the omentum, or both.

A hernia may be reducible or irreducible. It may become incarcerated, inflamed or strangulated.

A hernia is said to be strangulated when the herniated portion of the bowel or omentum is so tightly constricted as to cut off completely the circulation. The whole circumference of the knuckle of bowel may be involved, or only one side of the loop, so that the channel is not completely closed, or only part of the circumference of the bowel protrudes and becomes strangulated, as in Richter's hernia. In this case only a portion of the intestinal canal is involved, and the bowels often continue to act during the period of strangulation. The vermiform appendix occasionally becomes herniated, either by itself or with the bowel or omentum. I, myself, have seen two such occurring in the practice of others. Brieger (Archiv. für klin. Chir., Bd. 45, Hft. 4) reports twenty-two cases collected from different sources.

Whatever the contents of a hernial sac may be, the symptoms of strangulation are very much the same, though perhaps they differ much in degree.

The onset of strangulation is usually sudden, occurring generally while lifting or straining at stool, or doing some other exertion, especially when the thighs are flexed and adducted. Strangulation, however, may be gradual. But whether rapid or slow the effect is the same: the only difference is the length of time and the amount of constriction. The symptoms are generally so clear and well marked that a mistake in diagnosticating the condition is hardly possible; but occasionally cases are met with that are very puzzling. Because it is the most recent and standard work of surgery $I$ quote from the American Text-Book on Surgery, page 778, the following diagnostic signs: "The symptoms of strangulated intestine are always the same, whether the strangulation occurs in the abdomen or outside of it, viz., faintness, collapse, severe abdominal pain chiefly referred to the umbilical region, complete constipation, so that flatus ceases to pass, then vomiting first of the food, then of bile-stained fluid, and finally of fluid with a fecal odor. The tongue is dry and brown and the pulse rapid. In strangulated hernia there are in addition, at first pain at the seat of rupture, a greater degree of tension, the tumor having increased in size, dullness on percussion over the hernia, and absence of impulse on coughing, with the fact that the tumor is irreducible. If unrelieved the abdomen becomes much distended, retching is more frequent and thirst intense, hiccough sets in, the urine is much diminished in quantity and the patient gradually sinks from exhaustion in eight or nine days, though in very acute cases he may die in fortyeight hours. The bowel by this time has become gangrenous or there is a general peritonitis. With the onset of the gangrene there is often a cessation of the more urgent symptoms as vomiting and pain. Often the symptoms are not so severe as just described, especially in femoral hernia. In recent herniae the symptoms are more acute than in old herniæ and they are not so urgent when omentum is protruded with the bowel, or when the tumor has for some time been irreducible."

I thus quote at length because there are some important features to which I desire to call special attention. There are perhaps few surgical emergencies calling for more prompt and judicious action than strangulated hernia; and the question as to whether we shall operate at once or delay and palliate, is of supreme importance to the life of the patient. A few moments of hesitancy and doubt on the part of the surgeon, and the patient may be lost. So, gentlemen, if I appear a little tedious on this particular point I hope you will pardon me, for I can assure you I have felt keenly my responsibility as I have been brought to face these conditions.

The degree of collapse varies with the acuteness of the obstruction and the portion of the bowel obstructed. Thus, Mr. Treves states that the jejeunum with its richer blood and nerve supply would resent an injury more energetically than the lower parts of the intestines, where there is less functional activity. The collapse and vomiting are due largely to the impression made on the great abdominal nerve plexuses by the pinching of the nerve filaments in the gut.

Vomiting may come on early or late in the progress of the case. When it does occur it rarely ceases until the strangulation is relieved or the patient dies. At first the vomited matter consists of undigested food, then of bile and later there comes up great gushes of a dark brown muddy liquid of an intensely offensive fecal odor. No matter whether 
the vomiting appears early or late it is a symptom of grave omen. In a case of hernia in which any change has taken place in the condition of the tumor; such for example as an increase in size or tension with pain, and absence of expansile impulse, should be regarded as strangulated and treated as such. Do not wait for vomiting and other signs of intestinal obstruction. Vomiting is not a necessary condition of strangulation in the early stages and may not appear until gangrene is well established and septic peritonitis is present, and the powers of the patient so reduced as to often forbid a hope of recovery.

Pain may be very severe or it may be scarcely noticed. It may be at the seat of rupture and there may be wandering colicky pains all over the abdomen, but in nearly all cases there is a fixed dragging pain referred to the umbilicus.

Constipation is almost always present and is due, not necessarily to intestinal obstructions, but to the paralyzing effects on the visceral nerve supply. For we may have absolute constipation, and in fact generally do have constipation, where the herniated part consists only of omentum, or the appendix, or where the herniated part of the circumference of the bowel is included in the constriction and the canal is not obliterated, as in Richter's hernia, offering no mechanical impediment to the passage of fecal contents and gas; and further, we may have all the essential symptoms of strangulated hernia, such as vomiting, pain, constipation and collapse; but upon operating the sac is found to contain neither bowel nor omentum, only a little fluid, with no communication with the abdominal cavity; the operation, however, completely relieves the symptoms. While obstinate constipation is the rule, still Treves found three cases in fifty-three in which there was diarrhœa, and several that responded to laxatives.

The local symptoms are generally quite prominent. If the hernia is old and large and has been down many times, the size is likely increased and it may be fairly soft. Small strangulated herniæ are hard and tense, or if they contain omentum they feel doughy. Although the hernial tumor contains bowel, there may be dullness on percussion, owing to the small amount of air in the loop of the bowel, the thick walls, and to the fluid in the sac.

The impulse on coughing or straining is an important local feature and is of great diagnostic value; so much so indeed, that experienced surgeons have successfully calculated the nature of the contents of a hernial tumor by a careful estimation of the amount of expansion in the impulse. The impulse is principally due to the additional quantity of intestinal contents which is driven into the knuckle of gut by the act of coughing or straining. Of course it is very apparent that the amount and character of the impulse must be markedly influenced by the contents of the hernia. Thus in omental hernia, the expansion being due to the increased tension of the sac and to the sudden turgescence of the omental vessels, the impulse would naturally be relatively less in omental than in intestinal hernia. When strangulation occurs the contents of the sac are shut off from the abdomen and nothing more can enter; when the patient is made to cough the impulse is absent; provided, however, the constriction is situated as high at least as the internal abdominal ring. If the point of strangulation is below this all of that part of the hernia above will contribute toward an impulse. And it sometimes happens that all of the symptoms of strangulation are caused by a small knuckle of bowel being nipped at the extreme lower end of a long sac, thus leaving an excess of unstrangulated bowel. It is not uncommon to find in inguinal herniæ, long sacs extending down into the scrotum, divided into two or three compartments by two or more constricting bands or diaphragms. There is often a condition of free mobility in a strangulated hernia during coughing, especially if it be omental. The movements are, however, more of the nature of abrupt jumps and jerks in an up and down direction rather than expansile in character; that is to say, the tumor when the patient coughs not only rises under the hand but expands in size and is more marked in that part of the tumor lying near the ring. Again, when the strangulation is limited to the neck of the sac, although at the abdominal ring, the impulse may be present, provided, of course, the ring is not also concerned in the constriction. In very old and indurated masses of omentum, which completely fill the sac and block the external ring, even though it be not strangulated the expansile impulse may be absent.

As soon as possible after the diagnosis of strangulation is made it must be relieved, or the patient will surely die. Every moment increases the liability of gangrene in the bowel. The temporizing methods such as the application of heat and cold are applicable in only a very limited number of cases, and in fact they are generally used as aids to taxis. Applications of ice should be made only when the descent is recent, and even then not prolonged. In old persons with weak circulation, and in cases where the strangulation has existed for some time, the application of ice greatly favors gangrene. The application of heat can only be of value when an element of spasm exists and there is an irresistible desire to strain, as sometimes happens in large inguinal hernia.

Our means of reducing the strangulation is by taxis or herniotomy. Taxis is better adapted to inguinal than to femoral hernia, and to acquired than to congenital.

The patient should be etherized, and placed upon his back with his hips elevated and the thighs adducted and flexed upon the abdomen. The lower or external pillar of the ring blends with Poupart's ligament, to which is attached the fascia lata of the thigh, so that the position of the thigh affects the ring much. Thus extension and adduction of the thigh stretches the pillars and closes the ring, whereas flexion and adduction relaxes the pillars and opens the ring, relieves constriction and favors the return of the herniated viscera. Everything being ready the hands are thoroughly warmed, and while one hand firmly supports the neck of the hernia the other grasps the tumor and gently squeezes it, at the same time kneading it with a view of reducing its bulk. In manipulating the hernia great care must be used in making the pressure so that the whole surface of the finger pads and fingers are applied evenly, and the movements executed in a gentle, firm and regular manner. Avoid all forcible, unsteady and spasmodic manipulations; and above all avoid punching the finger tips into the parts, as they are admirably adapted for causing an unnecessary amount of bruising and possibly laceration of the gut. In inguinal hernia a slight downward pull is 
usually made to straighten it out and the pressure is directed upward and outward. In femoral hernia the pressure is made at first downward to bring it opposite the saphenous opening, and then backward and upward. Generally the gut slips in with a sudden gurgle which is quite characteristic, or if the omentum, there is a gradual change and a sensation of emptiness of the sac. In either case there is immediate relief of the symptoms. I mean, of course, provided the strangulation is relieved. But it sometimes happens that the sac is separated from its surroundings and pushed bodily in to the abdomen, with the contents still unreduced. This is termed reduction en bloc, in which case, although the tumor has disappeared the symptoms still persist. A trial of only a few moments is permissible during which manipulation may with safety be persisted in, in cases of apparent strangulation, no matter how gently it is applied. Even where a hernia is not strangulated prolonged manipulation may easily bring it about.

When properly applied taxis may be resorted to with propriety $(a)$ in ali cases seen very early, and the tension is not extreme; $(b)$ in hernial tumors dull on percussion, with omental or fluid contents, if the impulse is present: $(c)$ in cases where the symptoms are not urgent and where there has been no previous attempt made at reduction, and where the impulse is present. Taxis is more liable to succeed in inguinal than in femoral hernia, as the constricting tissues in the one are principally muscular, and in the other mainly ligamentous.

There are conditions in which taxis should not be resorted to at all in order to avert disaster. Here we have a loop of bowel distended with gas; rigid with vascular engorgement, constricted by a ring sometimes almost as sharp as a knife and over which the intestine acutely bulges in all directions, its vitality greatly impaired no fresh blood having entered it for hours; and under these circumstances and while in this condition an attempt is made to force it through the constriction. Can you imagine anything more unsurgical than this? Under these circumstances the bowel can be bruised or lacerated with the greatest ease. In fact, unless extreme gentleness and care are used some bruising will occur in the most favorable cases. In far advanced cases laceration may occur at the seat of stricture. Owing to the pressure exerted by the constricting band the bowel may already be partially eaten through by ulceration from within, or possibly it may be gangrenous at that point. Then very little force would be needed to complete the perforation. In other casess when the constricted part is not weakened by pathological changes instances are reported in which the gut was ruptured during taxis. In these cases the rent was not at the point of constriction, but on the distended portion of the bowel where the bulging was most prominent. In old irreducible hernia thee sometimes exist adhesions between the bowel and sac. If the adhesions themselves give way no harm follows; but they may be so strong and unyielding as to tear away some of the intestinal peritoneum in which event a partial laceration of the gut results, and is a serious accident if at all extensive. Another accident of taxis to which $I$ have already referred, is the reduction en massé of the sac together with its contents, the strangulation being unreduced. This has happened on slight manipulation, and most often occurs in recent herniæ, in which the sac is not adherent. Rupture of the sac may occur, the bowel protruding through the rent into the submucous tissue.

Even after the hernia has been reduced by taxis serious results may follow. If the intestine is inflamed at the time of the reduction; or, if owing to its long strangulation its vitality becomes so lowered as to allow its walls to be invaded by infective irritants, sloughing, perforation and peritonitis follows. A patient whose nutrition is bad may do well for three or four days, when, suddenly a small gangrenous patch separates and the patient sinks into a state of collapse and dies from shock.

Should taxis fail to return the hernia unstrangulated to the abdominal cavity we must proceed at once to the performance of herniotomy. I would earnestly recommend that arrangements be made before the patient is etherized to proceed without delay to the operation should taxis fail.

I will now report two cases upon which I have operated recently.

Case 1.-O. G., age 28, laborer. Has been ruptured for ten years. The tumor was not large and could always be easily reduced. Never wore a truss. A bout the middle of the forenoon of March 11, 1893, while lifting a piece of timber in a stooping posture, he suddenly felt something give way in his left side and he sank to the ground. Dr. Baird was called and found a large, painful, irreducible hernial tumor in the left groin extending into the scrotum. He was at once removed to St. Francis hospital where I saw him with Dr. Baird. The patient was vomiting persistently, and the pain was severe. The man, large and robust, was now pale, pinched and anxious looking, pulse rapid and he was in a cold clammy sweat. Collapse seemed imminent,so little time was used in trying to reduce by manipulation, but preparation for operation was at once begun. The patient was etherized, the abdomen was shaved and all the usual antiseptic precautions taken. I was ably assisted by Drs. Baird, Spencer and King. An incision some six inches in length was made directly over the tumor, and the sac exposed and opened. The sac contained considerable dark colored fluid and about two feet of the large bowel, including the sigmoid flexure. The strangulated loop was dark and rigid with congestion. The constricting band which was at the internal ring was cut slightly and an attempt made to return the bowel, trying first at one end of the loop and then at the other, but it could not be returned. I then enlarged the ring to the extent of perhaps half an inch, before $I$ could get the gut back into the abdominal cavity. Although the strangulation had existed for only about four hours its vitality was greatly impaired especially at one point where the constriction was firm, so that I hesitated somewhat about returning it. I did return it, however, and no bad results followed. I then dissected the sac free from the cord and other tissues and cut it off flush with the internal ring, after ligating it in sections with silkworm gut. The pillars were then sewn together with the same material, and I concluded the operation by doing McBurney's operation for radical cure. His bowels moved spontaneously on the second day and he made a prompt recovery. He is now at work in the woods and wears no truss.

Case 2.-I was called A pril 15, 1893, to see J. McG., age 27. He has had hernia several years. Was operated upon two years ago for strangulated hernia at which time the wound suppurated and he was in the hospital four months. He had worn a truss constantly. On this occasion, however, being somewhat under the influence of liquor he took off his truss. Within an hour his hernia came down and became strangulated. I saw him in the course of an hour and he was vomiting and suffering intense pain. I sent him to the hospital and tried taxis without an anæsthetic. I finally prepared for an operation, and etherized the patient, applied taxis for a few moments but failed. I sterilized and made an incision three inches long directly over the tumor which was on the right groin. The tissues were so much matted together as to hardly be distinguished. After much careful dissection the sac was opened. This structure was much thickened and opaque. The sac contained a small knuckle of bowel and a large piece of omentum, which was much bypertro 
phied, and adherent to the sac. After relieving the constriction at the ring, the bowel was returned quite readily but the omentum could not be reduced without much handling and bruising. I therefore ligated it off in small sections and cut off a piece as large as my hand, and returned the stump to the abdomen. I then did a McBurney operation for radical cure. The case did well. Had a spontaneous movement of the bowels on the third day. In about a week his temperature went up to 103 and on removing the dressing found a mural abscess in the lower pillar of the ring. This was opened and discharged pus, but closed in a few days and the case finally went on to recovery, the patient leaving the hospital in about six weeks.

There are a few interesting points suggested by these cases. Many surgeons advise to only slightly nick the constricting tissues; but it seems to me better surgery to make the incision free enough to allow the herniated part to be easily returned, than to make a slight cut so that the gut will have to be literally crowded back. For the bowel may be in such a critical condition that even slight handling may greatly lessen the patient's chances for recovery, or even precipitate a fatal result. Then, I think, the omentum is liable to complicate matters very much. If much injured it is liable to inflame and if infected set up a septic peritonitis. It may form adhesions near the ring and guide the bowel to that point, thus greatly favoring a return of the hernia. If it is much hypertrophied and does not return readily it had better be removed and in doing this considerable care is necessary. The omentum should never be tied off in one mass nor even in large sections owing to the liability to hemorrhage? A ligature may sufficiently constrict the tissues as to prevent bleeding at the time but the fat is soon absorbed from under the ligature and it gets loose and allows free hemorrhage. So it is necessary to ligate in small sections and be sure the bleeding is thoroughly stopped before returning to the abdomen. In neither of these cases could an impulse be made out and this influenced me very materially in promptly resorting to herniotomy. In fact, many surgeons contend that when the impulse is absent an attempt should not be made to reduce by manipulation if there exists any other symptoms of strangulation.

\section{REPORT OF CASES IN EVIDENCE OF AD- VANCED THEORY OF SURGICAL IMPU. NITY OF THE PERITONEAL VISCERA.}

Read in the section on Surgery and Anatomy, at the Forty-fourth Annual Meeting of the American Medical Association.

\section{BY JOHN E. LINK, M.D.}

TERRE IIAUTE, IND.

To the members of this Association I feel that I should make some explanation, if not apology, in presenting this work.

I am possibly somewhat differently situated from the most of the prominent workers of the Surgical Section, and therefore from the environment of my location am able to draw some conclusions at variance with the experience of the city and hospital surgeon and teacher.

There are, we may all know, two classes of surgeons radically different in their methods of doing good, the conservative and the bold or daring. And whilst I may have in some remarks before the Section as well as in printed articles seemed to imply that with regard to abdominal work I place myself with the latter, surely I do not wish to so stand. I am decidedly in favor of conservative means in all cases where delay is not hazardous, and surely I agree with Dr. Senn, when he says these cases of abdominal surgery are sometimes attended by the greatest of difficulty; and I emphasize this sentiment, and say none should venture but those thoroughly equipped with the essentials of knack, love of the good to be done and a thorough training incident to clinical teachings and experience. The fad of recording experiences, and the love of dramatic display have too often, I am sorry to say, within my own circle of observation, characterized the laparotomist. Removal of the ovaries for imaginary disease incident to neurotic constitution has been done, to my positive conviction, either through ignorance or viciousness in desire to make display of skill, where, as I have tried to show, no anatomical knowledge nor special surgical skill was required.

Hoping that I will be excused for these preliminary remarks, I will only add with regard to the misunderstanding between myself and Dr. Senn at Detroit, that I regret it, both for the sake of the interest that I entertain for my profession and the hazarding of a personal friendship.

That I am an advocate of cleanly and careful painstaking detail in surgery, when not interfering with an important principle, should stand without the saying. That Dr. Senn has done a grand work in this particular line is established, and I only wish to add my mite to the mountain rather than in any particular to tear it down.

What I expect or desire to accomplish in the experiments that I shall report, is to show that instead of the fine details of cleanliness, catgut, silkworm gut, kangaroo tendon ligature, Lembert stitch and all that, being an essential feature of success, there is a tolerance of tissue here that not only invites the skill of the surgeon but demands the surgical attention of the doctor, when traumatized; and that any and all lines of progress however desirable in improved technic are of far less importance than that of promptness in this emergency work, not only in cases of penetrating wounds which I hold stand first, but in many others: appendicitis not being less than second in importance, whether, with perforation and general diffusion throughout the peritoneal cavity, or threatening. I believe that all cases of uncomplicated perforation of the bowel, barring accident, can be saved.

I am not here to present complications in idiopathic nor traumatic cases. I leave that to Dr. Senn and his confrères. I am only here to prove peritoneal tolerance to outside entrance-that there are no organized enemies lurking in the normal atmosphere. They are only, or in the most part, in old hospital wards and we country doctors have only to keep our patients away from them, and "aseptic surgery" so-called need not distract a moment of our time and attention, so greatly needed in these penetrating wounds, appendicitis, intussusseption and obstruction of the lumen of the alimentary canal.

Antiseptic surgery got the credit at one time of making abdominal surgery practically safe. We are all, I think, now aware of the fact that the means so employed hazarded the life of the patient; but it has left a nucleus of thought in riddance of a pathological fallacy and popular superstition; and stands to abdominal surgery at least in about the same 